\title{
Thermal conductivity of silicon doped by phosphorus: ab initio study*
}

\author{
B. ANDRIYEVSKY ${ }^{1, \dagger}$, W. JANKE ${ }^{1}$, V.YO. STADNYK ${ }^{2}$, M.O. ROMANYUK ${ }^{2}$ \\ ${ }^{1}$ Faculty of Electronics and Computer Sciences, Koszalin University of Technology, 2 Śniadeckich Str., \\ PL-75-453, Koszalin, Poland \\ ${ }^{2}$ The Ivan Franko National University of Lviv, 8 Cyril and Methodius Str., UA-79005 Lviv, Ukraine
}

\begin{abstract}
An original approach to the theoretical calculations of the heat conductivity of crystals based on the first principles molecular dynamics has been proposed. The proposed approach exploits the kinetic theory of phonon heat conductivity and permits calculating several material properties at certain temperature: specific heat, elastic constant, acoustic velocity, mean phonon scattering time and coefficient of thermal conductivity. The method has been applied to silicon and phosphorus doped silicon crystals and the obtained results have been found to be in satisfactory agreement with corresponding experimental data. The proposed computation technique may be applied to the calculations of heat conductivity of pure and doped semiconductors and isolators.
\end{abstract}

Keywords: heat conductivity; molecular dynamics; ab initio calculations; phosphorus doped silicon crystals

\section{Introduction}

Silicon crystals doped with phosphorus, aluminium, boron, or arsenic are the materials frequently used in electronics. The problem of heat removal from the functional elements is crucial in the devices of high power electronics [1]. From this viewpoint, the thermal characteristics of corresponding materials and interfaces between them are therefore of great interest [2-8]. That is why the choice of adequate methods for the calculation of thermal conductivity and related values is significant for resolving different materials problems in this field.

Numerous theoretical techniques for determining lattice thermal conductivity have been reported in the literature and applied to a wide range of materials [9]. The molecular dynamics (MD) techniques may be divided into two main groups: (1) equilibrium and (2) non-equilibrium methods. In an equilibrium MD simulation, the system under

\footnotetext{
${ }^{*}$ This paper was presented at the $40^{\text {th }}$ International Microelectronics and Packaging (IMAPS Poland) Conference 2016, September 25 - 28, Wałbrzych - Wrocław.

†E-mail: bohdan.andriyevskyy@tu.koszalin.pl
}

investigation has a constant average temperature and an average heat flux of zero [9]. However, at each instant of time, a finite heat flux exists due to instantaneous fluctuations in temperature. The popular Green-Kubo method [10, 11], based on the general fluctuation-dissipation theorem [12], relates lattice thermal conductivity of a system to the time required for such fluctuations to dissipate.

In the non-equilibrium MD methods, a heat flux through the structure under study is applied and the temperature gradient that develops as a consequence of the imposed flux is determined. For example, in the version of the reverse nonequilibrium molecular dynamics (RNEMD), the heat flux is introduced by continuously transferring energy from a "cold" slab, located in the middle of the simulation cell, to the "hot" slabs, located at the ends of the simulation cell. This is accomplished by exchanging the velocities of the hottest atom in the cold slab with the coldest atom in the hot slab [13].

We present the results of ab initio equilibrium molecular dynamics study of the thermal conductivity in pure silicon and phosphorus doped silicon crystals. An original approach to calculation of the values related to the thermal conductivity 
of materials by using the ab initio molecular dynamics method (AIMD), based on the density functional theory (DFT), has been proposed. The method permits calculating the specific heat, acoustic velocity, mean phonon scattering time and coefficient of thermal conductivity of the materials at different temperatures. This approach uses the relation for the thermal conductivity coefficient derived from the kinetic theory of phonon propagation and is based on the calculations of standard molecular dynamics values: root meansquare atomic deviation, mean-square atomic displacement and spectral density of states. Results of the thermal conductivity characteristics in the obtained silicon and phosphorous doped silicon crystals have been found to be in good qualitative and satisfactory quantitative agreement with corresponding reference data.

\section{Method of computations}

According to the kinetic theory of phonon transport [14], the coefficient of thermal conductivity $\mathrm{K}$ is proportional to the mean phonon scattering time $\tau_{\mathrm{s}}$ :

$$
\kappa=\rho C v^{2} \tau_{s} / 3
$$

where $\rho$ is density of a material, $\mathrm{C}$ is the specific heat, and $\mathrm{v}$ is a mean acoustic phonon velocity. Three temperature dependent values, $\mathrm{C}, \mathrm{v}$, and $\tau_{\mathrm{s}}$ may be determined separately from the results of $\mathrm{ab}$ initio MD calculations.

The AIMD simulations of silicon have been performed in the framework of the DFT using the VASP package [15]. The projector augmentedwave (PAW) method with a plane waves cutoff energy of $300 \mathrm{eV}$ was employed [15, 16], together with the corresponding pseudopotentials. For the exchange and correlation terms, the gradient corrected Perdew-Burke-Ernzerhof (PBE) functional was used. Taking the relatively large diamond supercells $3 \times 3 \times 3$ of Si into account $(3 \mathrm{a}=16.29 \AA)$ only the $\Gamma$ point in the Brillouin zone was considered for geometry optimization. The optimized structures were used as an input for the computation of MD trajectories.
The AIMD calculations of Si were performed in the microcanonical NVE ensemble for different temperatures and initially optimized supercell $3 \times 3 \times 3$ at the temperature $\mathrm{T}=0 \mathrm{~K}$. Most results of the AIMD calculations have been obtained for the simulation time up to $15 \mathrm{ps}$ with the time step of 1.5 fs. For post-processing analyses, the post MD calculation program nMoldyn 3.0 was used [17].

Using the obtained MD-trajectories, the meansquare displacements (MSD) of atoms were calculated by the following relation:

$$
\sigma^{2}(t)=\frac{1}{N} \sum_{\alpha=1}^{N} w_{\alpha}\left\langle d_{\alpha}^{2}(t)\right\rangle
$$

where, $\left\langle d_{\alpha}^{2}(t)\right\rangle$ denotes the MSD for atoms of $\alpha$ type, $d_{\alpha}(t)=R_{\alpha}(t)-R_{\alpha}(0), w_{\alpha}$ is the weight coefficient and $t$ is the time. The velocity autocorrelation functions (VACF):

$$
F_{v v}(t)=\frac{1}{3 N} \sum_{\alpha=1}^{N} w_{\alpha}\left\langle v_{\alpha}(0) \cdot v_{\alpha}(t)\right\rangle
$$

and the spectral density of states (SDOS) - G( $\omega$ ) was calculated as Fourier transformation of $F_{v v}(t)$ :

$$
G(\omega)=\int_{0}^{\infty} \exp [-i \omega t] F_{v v}(t) d t
$$

According to the latter relation:

$$
G(0)=G_{0}=\int_{0}^{\infty} F_{v v}(t) d t=D
$$

that is the diffusion coefficient D of atoms [17]. In the case of relatively slow time dependent decrease of the amplitude of VACF (equation 3), corresponding to a large mean phonon scattering time $\tau_{\mathrm{s}}$, the time of MD simulation should be sufficiently large to obtain reliable values of $G_{0}(G$ at $\omega=0$ ) using the relation 5 .

The other relation often used for calculations of the diffusion coefficient D from MD studies is the Einstein-Smoluchowski relation for spatial atomic diffusion:

$$
\sigma^{2}=6 D t
$$


where $\mathrm{t}$ is the MD simulation time [18]. In the present study of solid silicon, where the observed diffusion of atoms is negligible, we have used the latter relation to obtain the mean phonon scattering time $\tau_{s}$ :

$$
\tau_{\mathrm{s}}=\frac{\sigma_{a}^{2}}{6 G_{0}}
$$

where the meaning of the value $\sigma_{\mathrm{a}}^{2}$ is explained in the following chapter. Thus, the key point of the present approach is the use of the EinsteinSmoluchowski relation 6 for diffusion (but with the special value $\sigma_{\mathrm{a}}^{2}$ ) to the calculation of phonon scattering time $\tau_{\mathrm{s}}$, which afterwards may be used for calculations of the corresponding thermal conductivity coefficient from the relation 1 .

\section{Results and discussion}

By performing two sets of MD calculation corresponding to two temperatures $T_{1}$ and $T_{2}$, we can evaluate the specific heat $\mathrm{C}$ :

$$
C=\frac{E_{2}-E_{1}}{m\left(T_{2}-T_{1}\right)}
$$

where $E_{1}$ and $E_{2}$ are the corresponding total energies and $\mathrm{m}$ is the mass of a crystal supercell.

The acoustic velocity $\mathrm{v}$ may be calculated from the elastic stiffness $\mathrm{c}$ and density $\rho$ of a crystal according to the know relation:

$$
v=\sqrt{\frac{c}{\rho}}
$$

To estimate the elastic stiffness $\mathrm{c}$ from the results of MD calculations one can use presentations of one atom energy in the forms of thermal energy (A), $3 \mathrm{k}_{\mathrm{B}} \mathrm{T} / 2$, and mechanical energy (B) (kinetic plus potential energies) of the corresponding effective oscillator, $\mathrm{kx}^{2} / 2+\mathrm{mv}^{2} / 2=\mathrm{kx}^{2}$. Here, $\mathrm{k}_{\mathrm{B}}$ and $\mathrm{k}$ are correspondingly Boltzmann and force constants. The characteristic atomic deviation $\mathrm{x}$ may be exchanged by the root mean square deviation (RMSD) R obtained from MD calculations at a temperature T:

$$
\frac{3 k_{\mathrm{B}} T}{2}=k R^{2}
$$

The force constant $\mathrm{k}$, if obtained, may be used for estimation of the elastic stiffness c, according to the relation:

$$
c A=k L
$$

resulted from the definitions of $\mathrm{k}$ and $\mathrm{c}$ values (A is an area and L is a length of the model parallelepiped containing one effective oscillator). For silicon diamond structure of the cubic symmetry we use $\mathrm{A}=\mathrm{L}^{2}$. For $\mathrm{L}$ value, one can take the nearest neighbor distance $\mathrm{d}_{\mathrm{Si}-\mathrm{Si}}=\mathrm{L}=2.35 \AA$, that is reasonable in terms of the oscillator model used.

Thus, on the basis of MD calculations performed at different temperatures there is a possibility to estimate the temperature dependent specific heat $\mathrm{C}(\mathrm{T})$, the elastic stiffness $\mathrm{c}(\mathrm{T})$ (equation 12) and the acoustic velocity $\mathrm{v}(\mathrm{T})$ (equation 13):

$$
c=\frac{3 k_{\mathrm{B}} T}{2 R^{2} L}
$$

$$
v=\sqrt{\frac{C}{\rho}}=\sqrt{\frac{3 k_{\mathrm{B}} T}{2 L R^{2} \rho}}
$$

The MSD $\sigma^{2}\left(\sigma^{2}=2 R^{2}\right)$ of $\mathrm{Si}$ as a function of temperature $\mathrm{T}$ is found to be close to the linear dependence with small positive nonlinearity:

$$
\begin{aligned}
\sigma^{2}(T)= & -3.77571 \cdot 10^{-6} \\
& +1.17433 \cdot 10^{-6} \cdot T+6.51337 \cdot 10^{-11} \cdot T^{2}
\end{aligned}
$$

where the magnitude of $\sigma^{2}$ is in $\mathrm{nm}^{2}$ units. According to the relation 10 and relation 12 , the detected small positive nonlinearity of the dependence $\sigma^{2}(\mathrm{~T}) 14$ is an evidence of small temperature decrease of the force constant $k$ and elastic stiffness $\mathrm{c}$ of silicon in the temperature range of $0 \mathrm{~K}$ to $1000 \mathrm{~K}$.

The temperature dependence $\mathrm{G}_{0}(\mathrm{~T})$ is a growing function with a clear positive nonlinearity (Fig. 1). Having the values $\sigma^{2}$ and $\mathrm{G}_{0}$ calculated, we have supposed that the corresponding mean phonon scattering time $\tau_{\mathrm{s}}$, necessary for calculation of the coefficient of thermal conductivity $\mathrm{K}$ (1), 
may be estimated from relation 7, which is similar to the Einstein-Smoluchowski relation for diffusion 6 . In the present case, no diffusion of $\mathrm{Si}$ atoms takes place and therefore the value $\mathrm{G}_{0}$ reflects the deviation of crystal structure from the perfect atomic distribution caused by the finite temperature and imperfections. Using the above supposition, we have calculated the dependence $\tau_{\mathrm{s}}(\mathrm{T})$ using the dependences $\sigma^{2}(T)$ and $G_{0}(T)$ obtained from the AIMD calculations. Afterwards, the corresponding temperature dependence of the thermal conductivity coefficient $k(T)$ was calculated using the relation 1 . We have found that the dependence $\kappa(T)$ is a decreasing function, similar to the experimental one, but the calculated absolute value $|\Delta \kappa / \Delta \mathrm{T}|$ is several times smaller than the corresponding experimental magnitude.

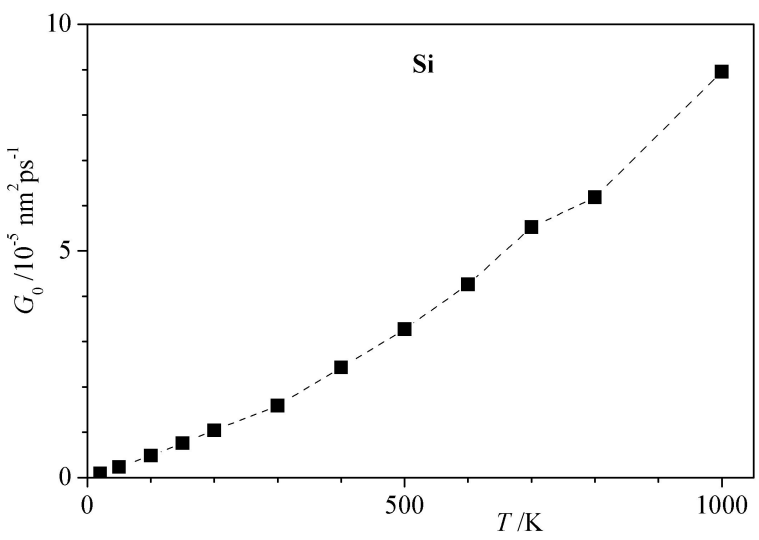

Fig. 1. Temperature dependence of $\operatorname{SDOS~}_{0}(\mathrm{~T})$ of silicon.

On the other hand, very close similarity of the calculated and experimental temperature dependences of $k(T)$ for silicon has been obtained when the temperature independent value of MSD, $\sigma_{\mathrm{a}}{ }^{2}$ was used for calculation of the scattering time $\tau_{\mathrm{s}}$ using the relation 7 . By comparing the calculation and experimental data on silicon we have found that the corresponding constant value of $\sigma_{\mathrm{a}}{ }^{2}$ should be taken as MSD at the temperature $\mathrm{T}=\mathrm{T}_{\mathrm{a}}$ corresponding to the acoustic frequency $v_{a}$ in accordance to the Debye relation $[19,20]$ :

$$
h v_{a}=k_{B} T_{a}
$$

The value $v_{\mathrm{a}}$, in turn, was taken close to the high frequency edge of the acoustic branch, $v_{\mathrm{a}}=7.4 \mathrm{THz} \leftrightarrow 246.8 \mathrm{~cm}^{-1}$, related to the SDOS G(v) (Fig. 2). This choice is grounded on the known fact that the phonons from the acoustic branches of the phonon dispersion relation $\omega(q)$ transfer mainly the heat in solids [21]. Especially, the acoustic velocity $v$, taken in the relation 1, is calculated as the group acoustic velocity $\mathrm{v}=(\mathrm{d} \omega / \mathrm{dq})_{\mathrm{q}=0}$ at $\mathrm{q}=0$. The velocities $\mathrm{d} \omega / \mathrm{dq}$ of the optical branches are much smaller. Thus, acoustic vibrations in the frequency range of $0 \mathrm{THz}$ to $7.4 \mathrm{THz}$ have been taken into account for estimation of the corresponding characteristic temperature $T_{a}$, which then has been used for calculation of the corresponding value $\sigma_{\mathrm{a}}{ }^{2}$. From the viewpoint of the known inverse proportional dependence of the mean phonon scattering time $\tau_{\mathrm{s}}$ and temperature $\mathrm{T}$ of a material and taking into account similar dependence of the inverse value $\mathrm{G}_{0}^{-1}(\mathrm{~T})$ obtained (Fig. 1), it looks reasonable that the value $\sigma_{\mathrm{a}}{ }^{2}$, used here for calculation of the phonon scattering time $\tau_{\mathrm{s}}$ by the relation 7 , should not be a temperature growing function, like the dependence $\sigma^{2}(\mathrm{~T})$.

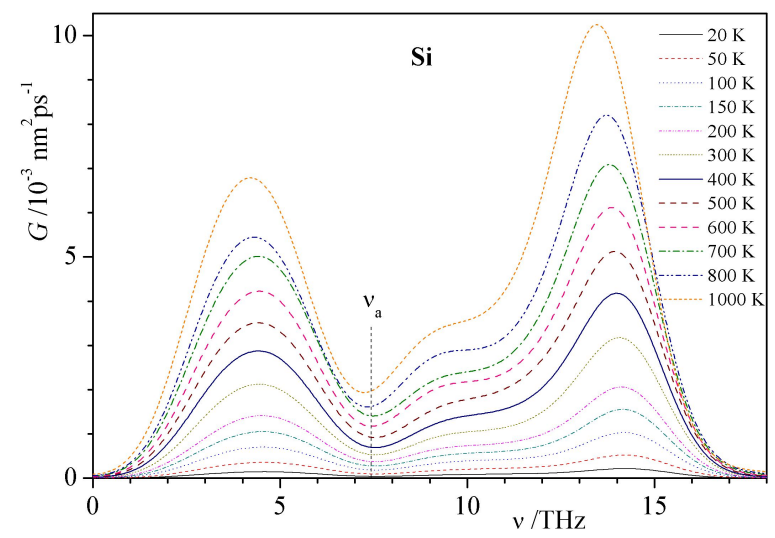

Fig. 2. SDOS as a function of frequency $v$ for silicon at different temperatures.

We have found that the temperature dependence of the coefficient of silicon thermal conductivity $\kappa(T)$ in the temperature range $300 \mathrm{~K}$ to $1000 \mathrm{~K}$ is determined mainly by the temperature dependence of the mean phonon scattering time $\tau_{\mathrm{s}}(\mathrm{T})$. The dependences on temperature of other values from the relation $1, \rho(\mathrm{T}), \mathrm{C}(\mathrm{T})$ and $\mathrm{v}(\mathrm{T})$, are much smaller in comparison to that for $\tau_{\mathrm{s}}(\mathrm{T})$. 
The distributions $\mathrm{G}(v)$ for different temperatures are similar (Fig. 2) and are in good agreement with the reference data for silicon [22]. However, a clear low frequency shift is observed with increasing temperature on the normalized dependences $\frac{\mathrm{G}(v)}{\mathrm{G}_{\max }}$ plot vs. $v$ (Fig. 3). The observed temperature dependent shifts of $\frac{\mathrm{G}(v)}{\mathrm{G}_{\max }}$ distributions indicate the decrease of the force constant $\mathrm{k}$ and elastic stiffness $c$ of the material because of the known relation for the corresponding resonance angular frequency, $\omega_{0}^{2}=\mathrm{k} / \mathrm{m}$. As a consequence, the elastic stiffness $\mathrm{c}$ is expected to be a slightly decreasing function of temperature $\mathrm{T}$. This expectation has been confirmed by calculations using the corresponding MSD data and the relation 10, relation 11 and relation 12 . We have found that the small decrease in the elastic stiffness $c_{11}$ from $\mathrm{c}_{11}{ }^{(300 \mathrm{~K})}=151 \mathrm{GPa}$ to $\mathrm{c}_{11}{ }^{(1000 \mathrm{~K})}=141 \mathrm{GPa}$ with temperature, is in a good agreement with the reference data [23] (difference is about $10 \%$ ). Taking into account also the temperature decrease of silicon density $\rho$ (near several percent) and the relation 9, one can expect almost $7 \%$ temperature decrease of the corresponding longitudinal acoustic velocity $v$ in the range of $300 \mathrm{~K}$ to $1000 \mathrm{~K}$.

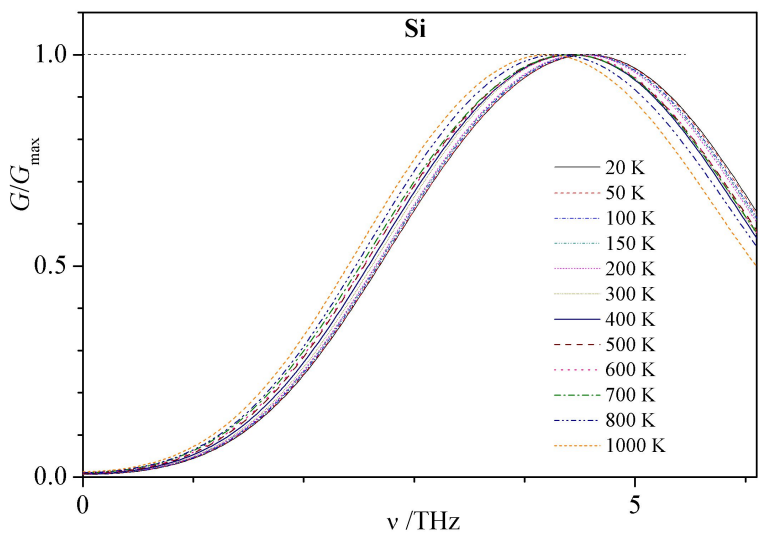

Fig. 3. Normalized SDOS $\mathrm{G} / \mathrm{G}_{\max }$ as a function of frequency $v$ for silicon at different temperatures.

The temperature dependence of specific heat $\mathrm{C}(\mathrm{T})$ of silicon at a constant volume $\mathrm{V}$, calculated with using the relation 8 , is found to be a slightly increasing function in the range of $300 \mathrm{~K}$ to $1000 \mathrm{~K}$, that is in satisfactory agreement (up to $30 \%$ ) with corresponding experimental observations for the specific heat at a constant pressure [24, 25].

According to the Debye relation 15 , the cutoff acoustic frequency of silicon $v_{\mathrm{a}}{ }^{(1)}=7.4 \mathrm{THz}$ corresponds to the temperature $\mathrm{T}_{\mathrm{a}}=355 \mathrm{~K}$. According to the relation 14 , this temperature $T_{a}$ corresponds to the value of MSD $\sigma^{2}\left(T_{a}\right)=\sigma_{a}^{2}=4.213 \cdot 10^{-4} \mathrm{~nm}^{2}$, which is used for the calculation by equation 7 of the temperature dependent mean phonon scattering time $\tau_{\mathrm{s}}{ }^{(1)}$ for $v_{\mathrm{a}}{ }^{(1)}=7.4 \mathrm{THz}$ (Fig. 4). For the cutoff acoustic frequency $v_{\mathrm{a}}{ }^{(2)}=6.0 \mathrm{THz}$ the value $\sigma_{\mathrm{a}}{ }^{2}$ is equal to $\sigma_{\mathrm{a}}^{2}=3.416 \cdot 10^{-4} \mathrm{~nm}^{2}$ and the corresponding mean phonon scattering time $\tau_{\mathrm{s}}{ }^{(2)}$ is smaller than $\tau_{\mathrm{s}}{ }^{(1)}$ for $v_{\mathrm{a}}{ }^{(1)}=7.4 \mathrm{THz}$ (Fig. 4). Taking into account the above analysis of the MD data for silicon and the relation 1 , one can conclude that the expected temperature changes of the thermal conductivity coefficient $K$ are caused by the corresponding changes, mainly of the mean phonon scattering time $\tau_{\mathrm{s}}$. This is confirmed by the temperature dependences $\tau_{\mathrm{s}}(\mathrm{T})$ (Fig. 4) calculated using the relation 7. The values of $\tau_{\mathrm{s}}$ decrease more than 4 times when temperature increases from $300 \mathrm{~K}$ to $1000 \mathrm{~K}$ (Fig. 4).

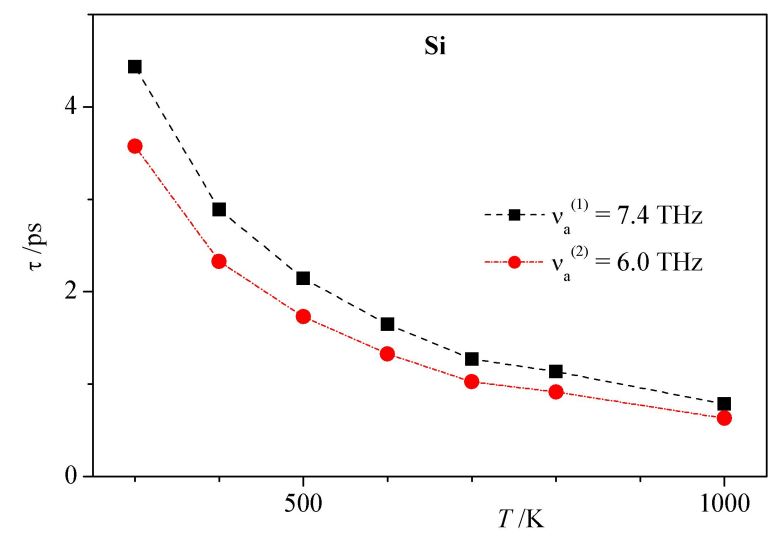

Fig. 4. Temperature dependences of mean phonon scattering time $\tau_{\mathrm{s}}(\mathrm{T})$ of silicon calculated for two values of cutoff frequency $v_{\mathrm{a}}^{(1)}=7.4 \mathrm{THz}$ and $v_{\mathrm{a}}^{(2)}=6.0 \mathrm{THz}$.

The calculated temperature dependence of the coefficient of thermal diffusivity $\mathrm{D}_{\tau}(\mathrm{T})$ :

$$
D_{\tau}=\frac{1}{3} v^{2} \tau_{s}
$$


obtained for pure silicon (Fig. 5) is found to be close to the reference data [26], that supports robustness of the present approach for calculations of the materials properties related to the thermal conductivity. On the basis of the thermal diffusivity data obtained for silicon (Fig. 5) and using the corresponding reference values for the specific heat $\left(\mathrm{C}=700 \mathrm{~J} \cdot \mathrm{kg}^{-1} \cdot \mathrm{K}^{-1}\right.$ [22]) one can calculate by the relation 1 the coefficients of thermal conductivity $\mathrm{K}_{1}=171.2 \mathrm{~W} \cdot \mathrm{m}^{-1} \cdot \mathrm{K}^{-1}\left(v_{\mathrm{a}}^{(1)}=7.4 \mathrm{THz}\right)$ and $\kappa_{2}=138.0 \mathrm{~W} \cdot \mathrm{m}^{-1} \cdot \mathrm{K}^{-1}\left(\nu_{\mathrm{a}}^{(1)}=6.0 \mathrm{THz}\right)$ at the temperature of $300 \mathrm{~K}$, which are close to the reference data [27].

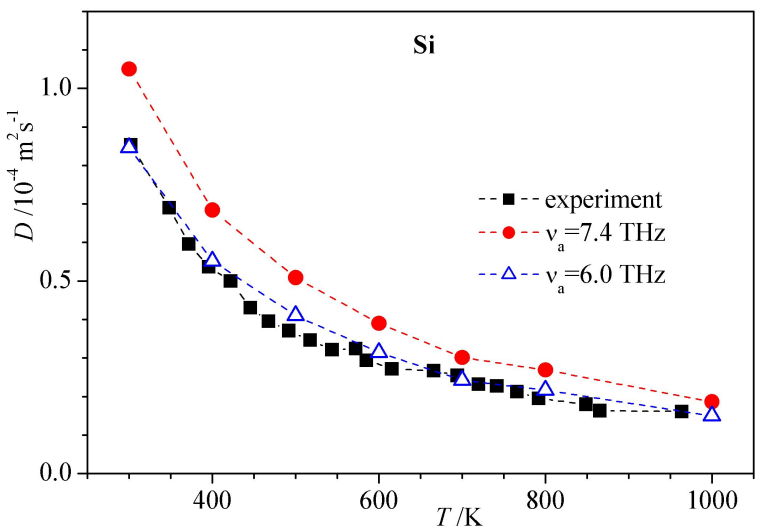

Fig. 5. Temperature dependences of diffusivity $D_{\tau}(T)$ for silicon: squares experiment [26], circles calculations for the cutoff frequency $v_{\mathrm{a}}=7.4 \mathrm{THz}$, triangles calculations for the cutoff frequency $v_{\mathrm{a}}=6.0 \mathrm{THz}$. Sound velocity $\mathrm{v}=8430 \mathrm{~ms}^{-1}$ [23] was taken for both calculated dependences.

We have applied the presented approach to the calculations of thermal conductivity characteristics of phosphorus doped silicon crystals. Five different concentrations of phosphorus atoms in five silicon based samples have been created and studied: 1, 3, 9,18 , and 27 silicon atoms were subsequently substituted by phosphorus in the $3 \times 3 \times 3$ supercell of silicon (216 atoms). The phosphorus atoms substituted the silicon ones in different places of the supercell, thus a homogeneous distribution of phosphorus has been ensured. Similar MD calculations (like in the case of the undoped silicon) have been performed for these five phosphorus doped silicon samples at two temperatures, $300 \mathrm{~K}$ and $700 \mathrm{~K}$, which enabled us to calculate the heat conductivity characteristics of these materials.

An increase of the phosphorus atoms concentration leads to several distinct changes in the frequency distributions of SDOS G(v) (Fig. 6): (1) the low frequency shifts of $G(v)$ dependences; (2) the opposite character of SDOS changes in the frequency regions of acoustic (around $4 \mathrm{THz}$ ) and optical (around $14 \mathrm{THz}$ ) vibration bands as a result of the change in relative phosphorus atoms concentration; (3) the increase of $\operatorname{SDOS~}_{0}(\mathrm{G}$ at $v=0)$. Similar changes take place for the temperature of $700 \mathrm{~K}$.

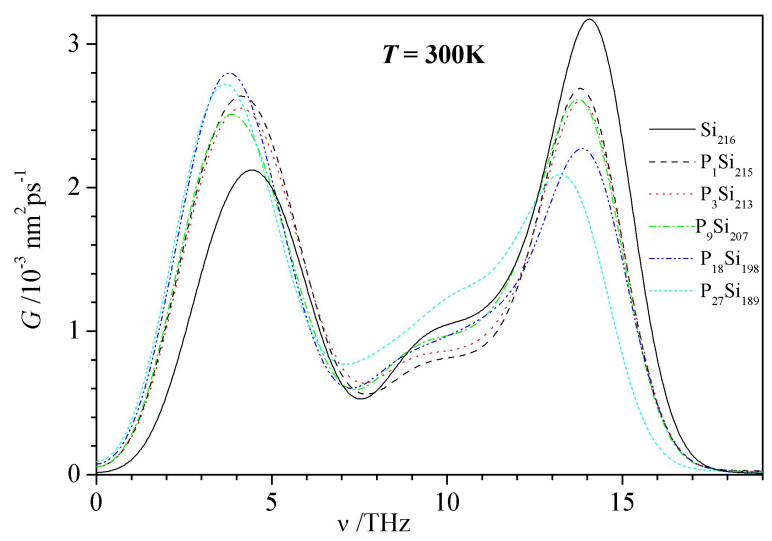

Fig. 6. SDOS $\mathrm{G}(v)$ of silicon $\left(\mathrm{Si}_{216}\right)$ and phosphorus doped silicon $\left(\mathrm{P}_{1} \mathrm{Si}_{215}, \mathrm{P}_{3} \mathrm{Si}_{213}, \mathrm{P}_{9} \mathrm{Si}_{207}\right.$, $\mathrm{P}_{18} \mathrm{Si}_{198}, \mathrm{P}_{27} \mathrm{Si}_{189}$ ) at the temperature of $300 \mathrm{~K}$.

An increase of the relative phosphorus concentration $\mathrm{n}_{\mathrm{Pr}}\left(\mathrm{n}_{\mathrm{Pr}}=\mathrm{N}_{\mathrm{P}} /\left(\mathrm{N}_{\mathrm{P}}+\mathrm{N}_{\mathrm{Si}}\right)\right)$ in the samples studied in the range of 0 to 0.125 leads to the increase of MSD $\sigma^{2}$ and SDOS $\mathrm{G}_{0}$ values (Fig. 7). The corresponding relative increase of SDOS, $\Delta \mathrm{G}_{0} / \mathrm{G}_{0}=0.65$, is more than three times larger than the relative increase of MSD, $\Delta\left(\sigma^{2}\right) / \sigma^{2}=0.19$. If we take into account the relation 7 and relation 1 , these results substantiate the decreasing dependence of the coefficient of thermal conductivity $\kappa\left(\mathrm{n}_{\mathrm{P}}\right)$ (Fig. 8). It is remarkable that pure silicon is characterized by much smaller values of $\mathrm{G}_{0}$ and moderately smaller $\sigma^{2}$ (Fig. 7) than similar values for the phosphorus doped silicon materials in the range of 0.0046 to 0.125 of $n_{P r}$. One therefore may claim that an increase in the phosphorus concentration $n_{P r}$ in the range of 0 to 0.0046 
should result in a large increase of $\mathrm{G}_{0}$ and moderate increase of $\sigma^{2}$ in the doped silicon in comparison to similar characteristics for the perfect structure of undoped $\mathrm{Si}$. As a result, the largest decrease of the dependences $\tau\left(\mathrm{n}_{\mathrm{Pr}}\right)$ and $k\left(\mathrm{n}_{\mathrm{Pr}}\right)$ is expected in the range of $n_{P r}$ from 0 to 0.0046 . Another feature of the obtained results is that the calculated value of $\mathrm{G}_{0}$ for the undoped silicon at the temperature of $1000 \mathrm{~K}$ (Fig. 1) is found to be close to the similar value for the heavily phosphorus doped silicon $\left(\mathrm{n}_{\mathrm{Pr}}=0.125\right)$ at the temperature of $300 \mathrm{~K}$ (Fig. 7). One, therefore, can compare two effects (temperature increase and phosphorus doping) leading to similar changes in the heat conductivity values.

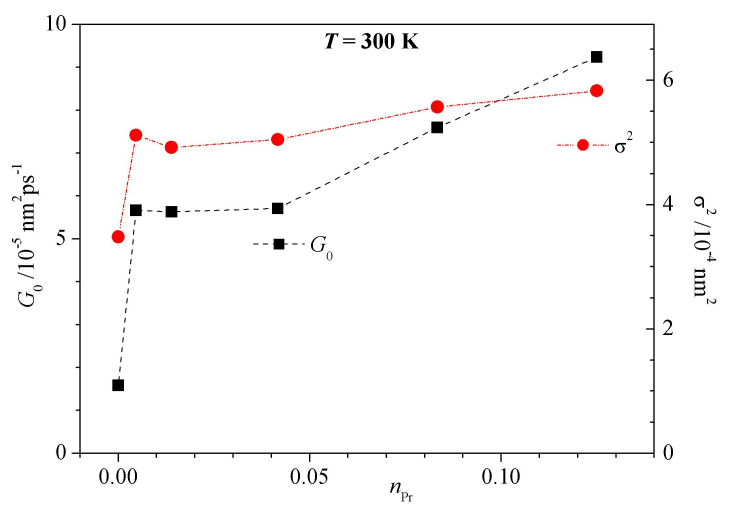

Fig. 7. Dependencies of SDOS $\mathrm{G}_{0}$ (left scale) and MSD $\sigma^{2}$ (right scale) of phosphorus doped silicon as a function of the relative phosphorus content $n_{\operatorname{Pr}}$ at the temperature of $300 \mathrm{~K}$.

The calculated dependence $\kappa\left(n_{\mathrm{P}}\right)$ (Fig. 8) agrees satisfactorily with the known reference data related to the study of the most heavily phosphorus doped silicon, $\kappa\left(\mathrm{n}_{\mathrm{P}}=5 \times 10^{20} \mathrm{~cm}^{-3}\right)=57 \mathrm{~W} \cdot \mathrm{m}^{-1} \cdot \mathrm{K}^{-1}[28]$. Our results are not in contradiction also with the heat conductivity studies of less heavily phosphorus doped Si [29, 30]. Besides, the obtained values of $\kappa$ (Fig. 8) may be comparable with the coefficients of thermal conductivity in the range of $38 \mathrm{~W} \cdot \mathrm{m}^{-1} \cdot \mathrm{K}^{-1}-29 \mathrm{~W} \cdot \mathrm{m}^{-1} \cdot \mathrm{K}^{-1}$ obtained for the surface doping of silicon by phosphorus in the doping range of $4 \cdot 10^{15} \mathrm{~cm}^{-2}$ to $10 \times 10^{15} \mathrm{~cm}^{-2}$ [31]

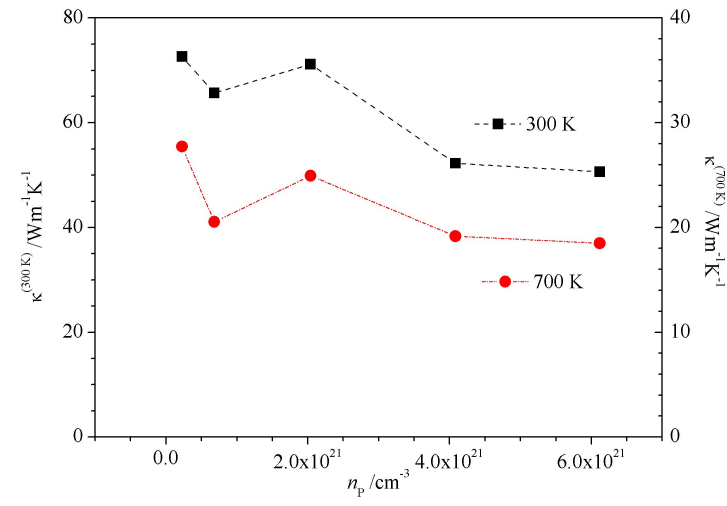

Fig. 8. Dependencies of the calculated coefficient of thermal conductivity of phosphorus doped silicon as a function of phosphorus doping concentration $\mathrm{k}\left(\mathrm{n}_{\mathrm{P}}\right)$ at the temperatures of $300 \mathrm{~K}$ (left scale) and $700 \mathrm{~K}$ (right scale). The open square point corresponds to the experimental thermal conductivity of the heavily phosphorus doped silicon at the temperature of $300 \mathrm{~K}$ [28].

\section{Conclusions}

1. The relation similar to the EinsteinSmoluchowski equation for atomic diffusion in solids may be applied for the calculation of mean phonon scattering time $\tau_{s}$, when the temperature independent mean squared displacement $\sigma_{\mathrm{a}}^{2}$ is used, the value of which is determined by the atomic displacements taking place in the propagation of acoustic waves in the studied material.

2. Using the results of $a b$ initio equilibrium molecular dynamics study of pure silicon and phosphorus doped silicon crystals obtained at different temperatures and phosphorus concentrations, the new approach for calculation of the mean phonon scattering time $\tau_{\mathrm{s}}$ and coefficient of thermal conductivity $K$ of the materials has been proposed.

3 . The temperature dependence of the coefficient of thermal conductivity $\kappa(T)$ of pure silicon and phosphorus content dependence $\kappa\left(n_{P}\right)$ of $p$-doped Si are determined mainly by the corresponding dependences of density of vibration states $\mathrm{G}_{0}(\mathrm{~T})$ and $\mathrm{G}_{0}\left(\mathrm{n}_{\mathrm{P}}\right)$ at zero vibration frequency $(v=0)$. 


\section{Acknowledgements}

The calculations were performed in the supercomputer centers ICM of Warsaw University in the framework of the project G26-3 and WCSS of Wrocław University of Science and Technology.

\section{References}

[1] Lidow A., Strydom J., Rooij de M., Reusch D., GaN Transistors for Efficient Power Conversion, Wiley, 2015.

[2] Borges R., Gallium nitride electronic devices for highpower wireless applications, Application Notes, RF Design, 2001, p. 72.

[3] Bernardoni M., Delmonte N., Menozzi R., CS Mantech Conference, Boston, USA, April 23 - 26, 2012.

[4] Perez J.A.F., Thermal Study of a GaN-Based HEMT, PhD Dissertation, University of Notre Dame Indiana, 2012.

[5] Visalli D., Optimization of GaN-on-Si HEMTs for High Voltage Applications, $\mathrm{PhD}$ Dissertation, Katholieke Universiteit Leuven, 2011.

[6] Fornetti F., Characterisation and Performance Optimisation of GaN HEMTs and Amplifiers for Radar Applications, PhD Dissertation, University of Bristol, 2010.

[7] MacFarlane D.J., Design and fabrication of AlGaN/GaN HEMTs with high breakdown voltages, $\mathrm{PhD}$ Dissertation, School of Engineering, University of Glasgow, 2014.

[8] Vitanov S., Palankovski V., Maroldt S., QUAY R., Solid-State Electron., 54 (2010), 1105.

[9] Stackhouse S., Stixrude L., Rev. Mineral. Geochem., 71 (2010), 253.

[10] Green M.S., J. Chem. Phys., 22 (1954), 398.

[11] Kubo R., J. Phys. Soc. Japan, 12 (1957), 570.

[12] Kubo R., Rep. Prog. Phys., 29 (1966), 255.

[13] Mullerplathe F.J., Chem. Phys., 106 (1997), 6082.
[14] Ziman J.M., Electrons and Phonons, Oxford University Press, 2001.

[15] Kresse G., Joubert D., Phys. Rev. B, 59 (1999), 1758.

[16] Blöchl P.E., Phys. Rev. B, 50 (1994), 17953.

[17] Róg T., Murzyn K., Hinsen K., KNeller G.R., J. Comput. Chem., 24 (2003), 657.

[18] Kaerger J., Grinberg F., Heituans P., Diffusion fundamentals, Leipzig University, 2005.

[19] Rohlf J.W., Modern Physics from A to Z, John Wiley \& Sons Inc, 1994.

[20] Blatt F.J., Modern Physics, McGraw-Hill, New York, 1992.

[21] Klemens P.G., Gell M., Mat. Sci. Eng. A, 245 (1998), 143.

[22] Tamura S., Shields J.A., Wolfe J.P., Phys. Rev. B, 44 (1991), 3001.

[23] Nikanorov S.P., Burenkov YU.A., Stepanov A.V., Sov. Phys. Solid State, 13 (1971), 2516.

[24] Okhotin A.S., Pushkarskil A.S., Gorbachev V.V., Thermophysical Properties of Semiconductors, "Atom" Publ. House, 1972. (in Russian).

[25] Desal P.D., J. Phys. Chem. Ref. Data, 15 (1986), 967.

[26] Shanks H.R., Maycock P.D., Sidles P.H., DANIElson G.C., Phys. Rev., 1305 (1963), 1743.

[27] Glassbrenner C.J., Slack G.A., Phys. Rev., 134 (1964), A1058.

[28] Lee Y., Hwang G.S., Phys. Rev. B, 86 (2012), 075202.

[29] Asheghi M., Kurab K., Kasnavi R., Goodson K.E., J. Appl. Phys., 91 (2002), 5079.

[30] JIN J.S., J. Mechan. Sci.Technol., 28 (2014), 2287.

[31] Xiel J., Lee C., Wang M.-F., LiU Y., Feng H., J. Micromech. Microeng., 19 (2009), 125029. 